\title{
External Ventricular Drainage before Endovascular Treatment in Patients with Aneurysmal Subarachnoid Hemorrhage in Acute Period: Its Relation to Hemorrhagic Complications
}

\author{
Yong Cheol Lim, $\mathrm{MD}^{1}$, Yu Shik Shim, $\mathrm{MD}^{2}$, Se-yang Oh, $\mathrm{MD}^{2}$, Myeong Jin Kim, $\mathrm{MD}^{3}$, \\ Keun Young Park, $\mathrm{MD}, \mathrm{PhD}^{4}$, Joonho Chung, $\mathrm{MD}, \mathrm{PhD}^{4,5}$ \\ ${ }^{1}$ Department of Neurosurgery, Ajou University Hospital, Ajou University College of Medicine, Suwon, Korea \\ ${ }^{2}$ Department of Neurosurgery, Inha University Hospital, Inha University College of Medicine, Incheon, Korea \\ ${ }^{3}$ Department of Neurosurgery, Gachon University Gil Medical Center, Incheon, Korea \\ ${ }^{4}$ Department of Neurosurgery, Stroke Center, Severance Hospital, Yonsei University College of Medicine, Seoul, Korea \\ ${ }^{5}$ Severance Institute for Vascular and Metabolic Research, Yonsei University College of Medicine, Seoul, Korea
}

Purpose: The purpose of this study was to report the authors' experience with external ventricular drainage (EVD) before endovascular treatment (EVT) in patients with acute aneurysmal subarachnoid hemorrhage (aSAH) and to investigate its relation to hemorrhagic complications.

Materials and Methods: Between March 2010 and December 2017, a total of 122 patients were recruited who had an aSAH, underwent EVT to secure the ruptured aneurysm, and had EVD performed within 72 hours of rupture. The pre-embo EVD group $(n=67)$ comprised patients who underwent EVD before EVT, and the post-embo EVD group ( $n=55)$ comprised those who underwent EVD after EVT.

Results: Overall, EVD-related hemorrhage occurred in 18 patients (14.8\%): six (8.9\%) in the pre-embo EVD group and $12(21.8 \%)$ in the post-embo EVD group ( $P=0.065)$. No rebleeding occurred between EVD and EVT in the pre-embo EVD group. Clinical outcomes at discharge did not differ significantly between groups $(P=0.384)$. At discharge, the final modified Rankin Scale score in patients who experienced pre-embo rebleeding was better in the pre-embo EVD group than in the post-embo EVD group ( $\mathrm{P}=0.041)$. Current use of an antiplatelet agent or anticoagulant on admission (odds ratio [OR], 2.928; 95\% confidence interval [Cl], 1.234-7.439; $\mathrm{P}=0.042)$ and stent use $(\mathrm{OR}, 2.430 ; 95 \% \mathrm{Cl}, 1.524-7.613 ; \mathrm{P}=0.047)$ remained independent risk factors for EVD-related hemorrhagic complications.

Conclusion: EVD before EVT in patients with aSAH in acute period did not increase the rate of rebleeding as well as EVD-related hemorrhagic complications. Thus, performing EVD before EVT may be beneficial by normalizing increased intracranial pressure. Especially in patients with rebleeding before the ruptured aneurysm is secured, pre-embo EVD may improve clinical outcomes at discharge

Key Words: Hydrocephalus; Subarachnoid hemorrhage; Intracranial aneurysm; Aneurysm, Ruptured

\section{Correspondence to: Joonho Chung, MD, PhD Department of Neurosurgery, Stroke Center, Severance Hospital, Severance Institute for Vascular and Metabolic Research, Yonsei University College of Medicine, 50-1 Yonsei-ro, Seodae- mun-gu, Seoul 03722, Korea \\ Tel: +82-2-2228-3391 \\ Fax: $+82-2-3461-9229$ \\ E-mail: ns.joonho.chung@gmail.com}

Received: July 30, 2018

Revised: August 2, 2018

Accepted: January 2, 2019

Copyright $\odot 2019$ Korean Society of Interventional Neuroradiology

This is an Open Access article distributed under the terms of the Creative Commons Attribution Non-Commercial License (http://creativecommons.org/licenses/by-nc/3.0) which permits unrestricted non-commercial use, distribution, and reproduction in any medium, provided the original work is properly cited. 


\section{INTRODUCTION}

Endovascular treatment (EVT) in patients with acute aneurysmal subarachnoid hemorrhage (aSAH) carries different risks than EVT of unruptured intracranial aneurysms, such as for acute hydrocephalus. External ventricular drainage (EVD) is one of the most important and common emergency procedures in neurosurgery mainly used to normalize or monitor intracranial pressure (ICP). ${ }^{1-3}$ Increased ICP should be normalized as soon as possible. Patients with aSAH have undergone EVT, but EVD has been suggested as a potential risk factor for hemorrhagic complications. ${ }^{4-7}$ When to perform EVD, before or after securing the ruptured aneurysm, remains controversial. EVD before EVT may increase rebleeding while EVD after EVT may delay normalizing increased ICP. Thus, the purpose of this study was to report the authors' experience with EVD before EVT in patients with acute aSAH and to investigate its relation to hemorrhagic complications.

\section{MATERIALS AND METHODS}

\section{Patient selection}

The institutional review board of our institute approved re- search protocols, and informed consent was waived. A prospective database contained a series of 1,393 patients with ruptured intracranial aneurysms treated by microsurgery or EVT between March 2007 and December 2017. Among them, EVD was performed for 468 patients. A total of 122 patients were included in this study, and all patients met the following criteria: 1) presence of aSAH, 2) underwent EVT to secure the ruptured aneurysm within 72 hours of rupture, and 3) EVD performed within 72 hours of rupture. Three hundred forty-six patients were excluded due to the following reasons: 1) patients underwent microsurgery for securing the ruptured aneurysm ( $n=136)$; 2) patients underwent EVT more than 72 hours after rupture $(n=55)$; 3 ) patients had a significant intracerebral hematoma due to the ruptured aneurysm requiring evacuation ( $n=42) ; 4)$ dissecting, fusiform, or blood blister-like aneurysm ( $n=60) ; 5)$ mycotic aneurysm ( $n=6) ; 6)$ traumatic pseudoaneurysm ( $n=9$ ); and 7 ) incomplete clinical or radiographic information $(n=38)$. The included patients were sorted into two groups: a pre-embo EVD group $(n=67)$ comprising patients who underwent EVD before EVT and a post-embo EVD group $(n=55)$ comprising those who underwent EVD after EVT.

Rebleeding was defined as sudden clinical deterioration with evidence of fresh blood on a computed tomography
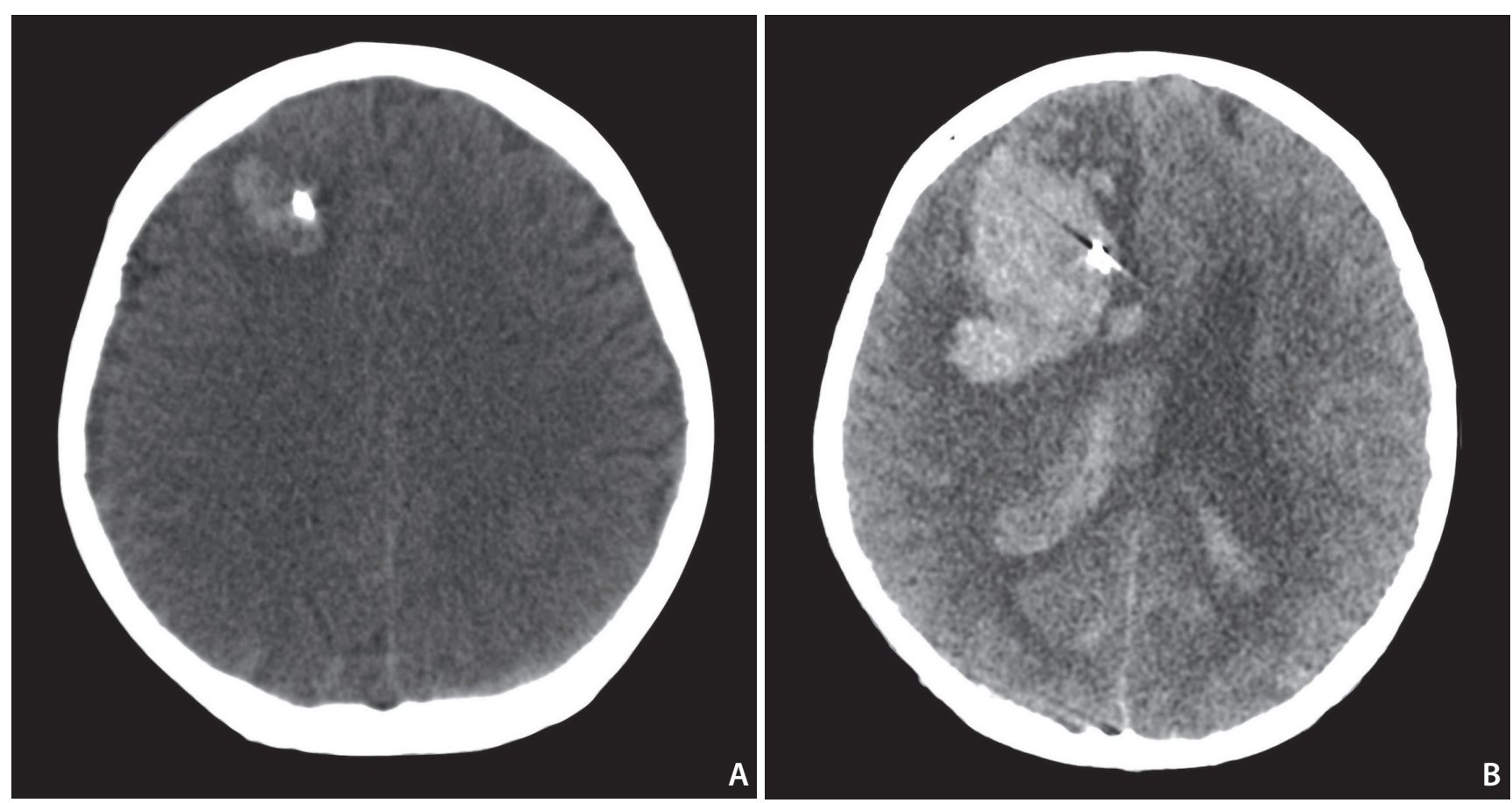

Fig. 1. External ventricular drainage-related hemorrhage along the ventricular catheter tract. (A) Small, asymptomatic hemorrhage and (B) symptomatic parenchymal hemorrhage requiring evacuation. 
(CT) scan in comparison with a previous scan or as evidence of contrast leaking on CT angiography (CTA) or digital subtraction angiography. Thromboembolic complications were defined as angiographic evidence of thrombosis during or at the end of EVT. Hemorrhagic complications were defined as a newly developed hemorrhage on CT or magnetic resonance image within 30 days after the procedure. EVD-related hemorrhagic complication was determined by two independent investigators. Regardless of clinical symptoms, hemorrhage was considered to be EVD-related if it occurred along the ventricular catheter tract after EVD placement (Fig. 1).

\section{External ventricular drainage and endovascular treatment}

After initial $\mathrm{CT}$ and $\mathrm{CTA}$ confirmed $\mathrm{aSAH}$, patients with acute hydrocephalus, intraventricular hemorrhage, or rapid deterioration of consciousness suggesting increased ICP underwent EVD. A hand drill with a 4.6-mm or 5.3-mm drill bit was used to perform EVD on Kocher's point or the supraorbital point in patients who were intubated with artificial ventilation under analgosedation using fentanyl, midazolam, morphine, or propofol. EVD was drained intermittently, to reach an ICP of $20 \mathrm{cmH}_{2} \mathrm{O}$ (slightly above normal ICP).

The decision to treat a ruptured aneurysm was made by agreement of microvascular neurosurgeons and neurointerventionists. All procedures were performed under general anesthesia with a standard approach from the common femoral artery. A 6- or 7-Fr guiding catheter was positioned in the internal carotid artery for anterior circulation aneurysms. A 5- or 6-Fr guiding catheter was positioned in the vertebral artery for posterior circulation aneurysms. Coils and stents were chosen according to the surgeon's preference. Heparin was not injected intravenously or subcutaneously but was instead mixed into saline flushes during the procedure. Therefore, the activated clotting time was not checked during the procedure. When stents were used, loading doses of clopidogrel (300-600 mg) and aspirin (200-400 mg) were given via a nasogastric tube after the femoral artery puncture or deploying stents. After the procedure, patients were prescribed $75 \mathrm{mg}$ clopidogrel daily for 3 months and $100 \mathrm{mg}$ aspirin daily for at least 12 months. During the procedure, $1 \mathrm{mg}$ of glycoprotein $\mathrm{llb} / \mathrm{llla}$ antagonist (tirofiban) was injected intra-arterially when the parent artery was occluded by a thromboembolism. Patients who underwent EVT without a stent did not take antiplatelet agents during or after the procedure.

\section{Clinical and radiographic outcomes}

Clinical outcomes and radiographic data were reviewed retrospectively. Patient factors (age, sex, hypertension, diabetes, smoking, dyslipidemia, coronary artery disease, antiplatelet regiment on admission, anticoagulant regiment on admission, initial Hunt-Hess grade, initial intracerebral hemorrhage, initial intraventricular hemorrhage, initial hydrocephalus, pre-embo rebleeding, and symptomatic vasospasm) and aneurysm characteristics (location [anterior or posterior circulation], aneurysm dome size [maximal diameter], neck size, aneurysm type [saccular or non-saccular], and stents use) were evaluated to identify risk factors for EVD-related hemorrhagic complications. Clinical outcomes were assessed with the modified Rankin scale (mRS) and evaluated by an independent investigator who was not involved in patient care. A favorable outcome was defined as mRS 0-2 and an unfavorable outcome was defined as mRS 3-6. The radiographic data were evaluated by two independent investigators.

\section{Statistical analysis}

All statistical analyses were performed with IBM SPSS Statistics version 22.0 (IBM, Armonk, NY, USA) in consultation with a biostatistician. Mann-Whitney $\mathrm{U}$ test was used for numeric variables. Chi-square test or Fisher exact test were used for nominal variables. Univariate analysis was performed to determine the association of EVD-related hemorrhage with other factors. Multivariate logistic regression was performed on variables with an unadjusted effect, and a P-value less than 0.10 on univariate analysis was used to determine risk factors independently associated with EVD-related hemorrhagic complications. A P-value less than 0.05 for a 95\% confidence interval (Cl) was considered statistically significant.

\section{RESULTS}

The radiographic characteristics and clinical outcomes of the 122 patients in both groups are shown in Table 1. Mean age was $56.5 \pm 12.4$ years in the pre-embo EVD group and $58.8 \pm 13.3$ years in the post-embo EVD group $(P=0.527)$. Initial Hunt-Hess grade differed significantly between groups $(P=0.034)$. The pre-embo EVD group had more patients with a poor grade. Nineteen patients (28.4\%) in the pre-embo EVD group and eight (14.5\%) in the post-embo EVD group had initial intracerebral hemorrhage $(P=0.094)$. Rebleeding before EVT seemed more common in the pre-embo EVD 
group (28.4\%) than in the post-embo EVD group (41.8\%), but the difference was not statistically significant $(P=0.120)$. Anterior circulation aneurysms were more common than posterior circulation aneurysms. Mean aneurysm size and neck size were $6.5 \pm 1.5$ and $3.1 \pm 0.9$, respectively, and did not differ between groups. Overall, EVD-related hemorrhage oc- curred in 18 patients (14.8\%): six (8.9\%) in the pre-embo EVD group and 12 (21.8\%) in the post-embo EVD group ( $\mathrm{P}=0.065)$. Three patients (one in the pre-embo EVD group and two in the post-embo EVD group) had symptomatic parenchymal hemorrhage requiring surgical evacuation. The other patients had small, asymptomatic hemorrhage along the ven-

Table 1. Clinical and radiographic characteristics of two groups

\begin{tabular}{|c|c|c|c|c|}
\hline & Total $(n=122)$ & Pre-embo EVD $(n=67)$ & Post-embo EVD $(n=55)$ & P-value \\
\hline Age (years) & $57.2 \pm 12.9$ & $56.5 \pm 12.4$ & $58.8 \pm 13.3$ & $0.527^{*}$ \\
\hline Female & $92(75.4)$ & $49(73.1)$ & $43(78.2)$ & 0.674 \\
\hline Hypertension & $44(36.1)$ & $27(40.3)$ & $17(30.9)$ & 0.286 \\
\hline Diabetes & $24(19.7)$ & $12(17.9)$ & $12(21.8)$ & 0.428 \\
\hline Smoking & $22(18.0)$ & $10(14.9)$ & $12(21.8)$ & 0.327 \\
\hline Dyslipidemia & $25(20.5)$ & $15(22.4)$ & $10(18.2)$ & 0.452 \\
\hline Coronary artery disease & $9(7.4)$ & $5(7.5)$ & $4(7.3)$ & 0.893 \\
\hline Antiplatelet agent on admission & $15(12.3)$ & $9(13.4)$ & $6(10.9)$ & 0.734 \\
\hline Anticoagulant on admission & $5(4.1)$ & $3(4.5)$ & $2(3.6)$ & 0.682 \\
\hline Initial Hunt-Hess grade & & & & 0.034 \\
\hline Grade 1 & $0(0)$ & $0(0)$ & $0(0)$ & \\
\hline Grade 2 & $2(1.6)$ & $0(0)$ & $2(3.6)$ & \\
\hline Grade 3 & $16(13.1)$ & $5(7.5)$ & $11(20.0)$ & \\
\hline Grade 4 & $69(56.6)$ & $35(52.2)$ & $34(61.8)$ & \\
\hline Grade 5 & $35(28.7)$ & $27(40.3)$ & $8(14.5)$ & \\
\hline Initial intracerebral hemorrhage & $27(22.1)$ & $19(28.4)$ & $8(14.5)$ & 0.094 \\
\hline Initial intraventricular hemorrhage & $88(72.1)$ & $46(68.7)$ & $42(76.4)$ & 0.152 \\
\hline Initial hydrocephalus & $55(45.1)$ & $32(47.8)$ & $23(41.8)$ & 0.314 \\
\hline Pre-embo rebleeding & $42(34.4)$ & $19(28.4)$ & $23(41.8)$ & 0.120 \\
\hline Aneurysm location & & & & 0.296 \\
\hline Anterior circulation & $95(77.9)$ & $54(80.6)$ & $41(74.5)$ & \\
\hline Posterior circulation & $27(22.1)$ & $13(19.4)$ & $14(25.5)$ & \\
\hline Aneurysm size (mm) & $6.5 \pm 1.5$ & $6.4 \pm 1.3$ & $6.8 \pm 1.6$ & $0.312^{*}$ \\
\hline Neck size (mm) & $3.1 \pm 0.9$ & $3.4 \pm 1.1$ & $2.9 \pm 1.2$ & $0.384^{*}$ \\
\hline Aneurysm type & & & & 0.622 \\
\hline Saccular & $95(77.9)$ & $51(76.1)$ & $44(80.0)$ & \\
\hline Non-saccular & $27(22.1)$ & $16(23.9)$ & $11(20.0)$ & \\
\hline Stents use & $40(32.8)$ & $24(35.8)$ & $16(29.1)$ & 0.272 \\
\hline EVD-related hemorrhage & $18(14.8)$ & $6(8.9)$ & $12(21.8)$ & 0.065 \\
\hline Symptomatic vasospasm & $22(18.0)$ & $12(17.9)$ & $10(18.2)$ & 0.848 \\
\hline Outcomes at discharge & & & & 0.384 \\
\hline Favorable (mRS 0-2) & $32(26.2)$ & $19(28.4)$ & $13(23.6)$ & \\
\hline Unfavorable (mRS 3-6) & $90(73.8)$ & $48(71.6)$ & $42(76.4)$ & \\
\hline
\end{tabular}

Values are presented as mean \pm standard deviation or number (\%).

EVD, external ventricular drainage; mRS, modified Rankin scale.

${ }^{*}$ According to Student t-tests. 
tricular catheter tract. No rebleeding occurred between EVD and EVT in the pre-embo EVD group.

Logistic regression adjusted for age and sex showed that antiplatelet or anticoagulant use on admission (odds ratio
[OR], 2.928; 95\% Cl, 1.234-7.439; $\mathrm{P}=0.042)$ and stent use (OR, 2.430; 95\% Cl, 1.524-7.613; $\mathrm{P}=0.047$ ) remained independent risk factors for EVD-related hemorrhagic complications (Table 2). Aneurysm neck size, aneurysm type, and EVD timing

Table 2. Risk factors for EVD-related hemorrhagic complications

\begin{tabular}{|c|c|c|c|c|}
\hline & \multicolumn{2}{|c|}{ Unadjusted } & \multicolumn{2}{|c|}{ Adjusted } \\
\hline & OR $(95 \% \mathrm{Cl})$ & P-value & OR (95\% Cl) & P-value \\
\hline \multicolumn{5}{|l|}{ Age in years } \\
\hline$<57$ & 1 & & 1 & \\
\hline$\geq 57$ & $1.305(0.438-4.226)$ & 0.659 & $1.156(0.461-3.594)$ & 0.713 \\
\hline \multicolumn{5}{|l|}{ Gender } \\
\hline Female & 1 & & 1 & \\
\hline Male & $1.219(0.778-5.061)$ & 0.538 & $1.288(0.647-4.871)$ & 0.622 \\
\hline Hypertension & $2.280(0.635-4.782)$ & 0.636 & & \\
\hline Diabetes & $1.587(0.716-3.344)$ & 0.486 & & \\
\hline Smoking & $0.930(0.308-2.904)$ & 0.585 & & \\
\hline Dyslipidemia & $1.262(0.648-3.742)$ & 0.725 & & \\
\hline Coronary artery disease & $1.443(0.840-4.622)$ & 0.147 & & \\
\hline Antiplatelet or anticoagulation & $3.142(1.483-6.570)$ & 0.038 & $2.928(1.234-7.439)$ & 0.042 \\
\hline \multicolumn{5}{|l|}{ Use on admission } \\
\hline \multicolumn{5}{|l|}{ Initial Hunt-Hess grade } \\
\hline Good (grade 1, 2, or 3) & 1 & & & \\
\hline Poor (grade 4 or 5) & $2.794(0.89-6.737)$ & 0.108 & & \\
\hline Initial intracerebral hemorrhage & $0.741(0.336-1.087)$ & 0.214 & & \\
\hline Initial intraventricular hemorrhage & $1.535(0.827-3.134)$ & 0.369 & & \\
\hline Initial hydrocephalus & $0.893(0.552-1.213)$ & 0.301 & & \\
\hline \multicolumn{5}{|l|}{ Aneurysm location } \\
\hline Anterior circulation & 1 & & & \\
\hline Posterior circulation & $2.008(0.921-4.831)$ & 0.223 & & \\
\hline \multicolumn{5}{|l|}{ Aneurysm size (mm) } \\
\hline$<6.5$ & 1 & & & \\
\hline$\geq 6.5$ & $0.826(0.783-1.093)$ & 0.553 & & \\
\hline \multicolumn{5}{|l|}{ Neck size (mm) } \\
\hline$<3.1$ & 1 & & & \\
\hline$\geq 3.1$ & $1.569(0.920-2.344)$ & 0.098 & $1.785(0.823-4.311)$ & 0.236 \\
\hline \multicolumn{5}{|l|}{ Aneurysm type } \\
\hline Saccular & 1 & & 1 & \\
\hline Non-saccular & $2.779(0.997-4.540)$ & 0.052 & $2.252(0.854-7.785)$ & 0.089 \\
\hline Stent use & $2.846(1.815-5.906)$ & 0.041 & $2.430(1.524-7.613)$ & 0.047 \\
\hline \multicolumn{5}{|l|}{ EVD timing } \\
\hline Pre-embo & 1 & & 1 & \\
\hline Post-embo & $2.797(0.925-7.503)$ & 0.088 & $2.150(0.812-8.857)$ & 0.154 \\
\hline
\end{tabular}

EVD, external ventricular drainage; $\mathrm{Cl}$, confidence interval; $\mathrm{OR}$, odds ratio. 
(pre- vs. post-embo) had P-values of 0.098, 0.052, and 0.088, respectively, in a simple logistic regression but were not statistically significant after adjustment $(P=0.236,0.089$, and 0.154 , respectively). EVD timing (pre- vs. post-embo) was not a risk factor for EVD-related hemorrhagic complications, suggesting that pre-embo EVD did not increase EVD-related hemorrhagic complications compared to post-embo EVD.

Clinical outcomes at discharge did not differ significantly between groups ( $P=0.384$ ). Overall, 32 patients (26.2\%) had favorable outcomes and 90 (73.8\%) had unfavorable outcomes at discharge. Clinical outcome at discharge, adjusted for age, sex, and initial Hunt-Hess grade, in patients who had pre-embo rebleeding (19 in the pre-embo EVD group and 23 in the post-embo EVD group) were better in the pre-embo EVD group than in the post-embo EVD group ( $P=0.041$; Fig. 2).

\section{DISCUSSION}

In our small series, EVD-related hemorrhagic complications occurred in $14.8 \%$ of patients. Although EVD-related hemorrhage seemed more common in post-embo EVD (21.8\%) than in pre-embo EVD (8.9\%), the difference was not statistically significant $(P=0.065)$. In addition, no rebleeding occurred due to pre-embo EVD in our series, suggesting that pre-embo EVD did not increase hemorrhagic complications, such as rebleeding or EVD-related hemorrhage. EVD-related hemorrhage rates of $15-20 \%$ have been reported in patients with aSAH. ${ }^{8,9}$ Data regarding the risk of rebleeding after EVD in patients with an unprotected aneurysm remains conflicting. Hasan et al. ${ }^{6}$ prospectively studied 473 patients and reported that the rebleeding rate within 12 days of aSAH was significantly higher in patients with EVD (43\%) than in those without (15\%, $P=0.025)$. However, the authors did not provide information on EVD timing. ${ }^{6}$ Paré et al. ${ }^{7}$ reported on a cohort of 128 patients with aSAH. Patients with EVD (30\%) had a higher incidence of rebleeding than those without (8\%, $\mathrm{P}<0.006$ ). A retrospective study of 304 patients with aSAH demonstrated no difference in rebleeding rates among patients with (4.4\%) or without EVD (5.4\%). ${ }^{10}$ Another study also concluded that EVD was not associated rebleeding in patients with aSAH. ${ }^{11}$ Hellingman et al. ${ }^{5}$ reported no difference between the incidence of rebleeding with preoperative EVD (21\%) and controls (21\%) in a study of 546 prospectively recruited patients with aSAH. However, previous studies did not account for time gap between EVD and aneurysm treatments. Additionally, the interval between onset of aSAH and EVD was vague. In the present study, thus, we tried to assess the safety of EVD placement before securing ruptured aneurysms in the acute period and to emphasize that controlling increased ICP is more important in such patients to improve clinical outcomes. In the same context, we found that clinical outcomes at discharge were better in the pre-embo EVD group than in the post-embo EVD group, suggesting that pre-embo EVD improved clinical outcomes by controlling ICP. Securing a ruptured aneurysm before EVD in patients with increased ICP may delay normalizing ICP and induce brain damage.

Among 468 patients who underwent EVD, pre-embo rebleeding occurred in 45 patients (9.6\%). In our practice, we tend to perform EVT rather than microsurgery in patients with pre-embo rebleeding, including 42 of the 45 patients in
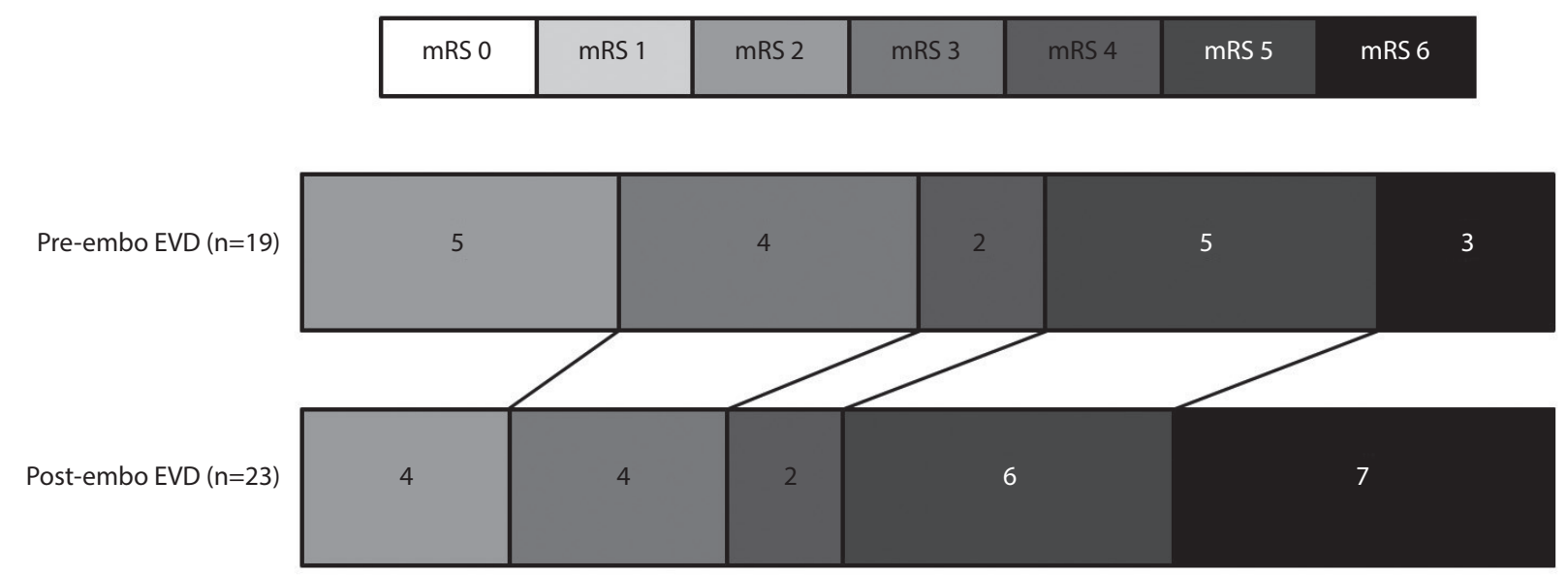

Fig. 2. Clinical outcomes (mRS) for two groups of patients who experienced pre-embo rebleeding at discharge $(P=0.041)$. $m R S$, modified Rankin scale; EVD, external ventricular drainage. 
the present study. A good initial Hunt-Hess grade was more common in the post-embo EVD group ( $P=0.034)$ than in the pre-embo EVD group because we performed one-staged coiling immediately followed by EVD if rebleeding occurred.

Patients who undergo EVT for aSAH need antiplatelet agents, anticoagulant agents, or both to prevent potential thromboembolic complications. Consequently, hemorrhagic complications can occur if patients with acute aSAH require additional surgical procedures after EVT. We identified two risk factors for EVD-related hemorrhagic complications: stent use and antiplatelet or anticoagulant use on admission. These risk factors are not surprising because physicians who treat intracranial aneurysms know that they are related to bleeding tendency. If stent use is considered to require antiplatelet use, both risk factors have same mechanism to provoke hemorrhagic complications. In our previous study of stent-assisted coil embolization in patients with acute aSAH, EVD was the only independent risk factor for periprocedural complications in a multivariate logistic regression. ${ }^{12}$ Bruder et al. ${ }^{13}$ analyzed the rate of EVD-related hemorrhage in patients with aSAH with or without artificial anticoagulation therapy during aneurysm treatment. EVD-related hemorrhage occurred in $12 \%$ of patients with aSAH. The rate of EVD-related hemorrhage in patients with additional anticoagulation after securing aneurysm was $24 \%$ versus $8 \%$ in patients without anticoagulation $(\mathrm{P}<0.001)^{13}$ In our practice, heparin was not injected intravenously or subcutaneously but was instead mixed into saline flushes during the procedure because we felt that hemorrhagic complications were more fatal than thromboembolic complications in patients with aSAH. If thromboembolism occurred during the procedure, we injected up to $1 \mathrm{mg}$ of glycoprotein Ilb/Illa antagonist (tirofiban) intra-arterially.

This study has several limitations. It is a retrospective study and included a small number of patients, which lowers the power to identify the true incidence of EVD-related complications. The individual response to each antiplatelet agent was not evaluated. A study on coronary intervention suggested that $30 \%$ of patients have relative clopidogrel resistance. ${ }^{14}$ Thus, a subset of patients probably over- or under-responded to antiplatelet agents. Comparison of clinical outcomes between groups of patients with aSAH should be adjusted for well-known predisposing or risk factors. In the present study, clinical outcomes for two groups of patients who experienced pre-embo rebleeding in Fig. 2 was adjusted for age, sex, and initial Hunt-Hess grade. However, comparison of overall clinical outcomes at discharge between groups in Table 1 was not adjusted for those factors. It might show different results if adjusted because the initial Hunt-Hess grade was significantly different between two groups. Finally, we did not assess activity of the coagulation cascade. An individual's potential to generate thrombin may correlate closely with a hypercoagulable state, which may have supported our results. Therefore, monitoring changes in prothrombin time, partial thromboplastin time, or thrombin generation ${ }^{15}$ may help predict EVD-related hemorrhagic complications.

\section{CONCLUSION}

EVD before EVT in patients with aSAH in the acute period did not increase the rate of rebleeding or EVD-related hemorrhagic complications. Thus, performing EVD before EVT may be beneficial to normalize increased ICP. Especially in patients with rebleeding before a ruptured aneurysm is secured, pre-embo EVD may improve clinical outcomes at discharge.

\section{Acknowledgments}

This study was supported by a faculty research grant of Yonsei University College of Medicine (6-2017-0130) and the Korean Society of Interventional Neuroradiology (KSIN) research grant 2017.

\section{REFERENCES}

1. Gigante P, Hwang BY, Appelboom G, Kellner CP, Kellner MA, Connolly ES. External ventricular drainage following aneurysmal subarachnoid haemorrhage. Br J Neurosurg 2010;24:625632

2. Fountas KN, Kapsalaki EZ, Machinis T, Karampelas I, Smisso HF, Robinson JS. Review of the literature regarding the relationship of rebleeding and external ventricular drainage in patients with subarachnoid hemorrhage of aneurysmal origin. Neurosurg Rev 2006;29:14-18; discussion 19-20

3. Bratton SL, Chestnut RM, Ghajar J, McConnell Hammond FF, Harris OA, Hartl R, et al. VII. Intracranial pressure monitoring technique. J Neurotrauma 2007;24 Suppl 1:S-45-S-54

4. Gardner PA, Engh J, Atteberry D, Moossy JJ. Hemorrhage rates after external ventricular drain placement. J Neurosurg 
2009;110:1021-1025

5. Hellingman CA, van den Bergh WM, Beijer IS, van Dijk GW, Algra A, van Gijn J, et al. Risk of rebleeding after treatment of acute hydrocephalus in patients with aneurysmal subarachnoid hemorrhage. Stroke 2007;38:96-99

6. Hasan D, Vermeulen M, Wijdicks EF, Hijdra A, van Gijn J. Management problems in acute hydrocephalus after subarachnoid hemorrhage. Stroke 1989;20:747-753

7. Paré $L$, Delfino R, Leblanc R. The relationship of ventricular drainage to aneurysmal rebleeding. J Neurosurg 1992;76:422427

8. Ko JK, Cha SH, Choi BK, Lee Jl, Yun EY, Choi CH. Hemorrhage rated associated with two methods of ventriculostomy: external ventricular drainage vs. ventriculoperitoneal shunt procedure. Neurol Med Chir (Tokyo) 2014;54:545-551

9. Scholz C, Hubbe U, Deininger M, Deininger MH. Hemorrhage rates of external ventricular drain (EVD), intracranial pressure gauge (ICP) or combined EVD and ICP gauge placement within $48 \mathrm{~h}$ of endovascular coil embolization of cerebral aneurysms. Clin Neurol Neurosurg 2013;115:1399-1402
10. Mclver Jl, Friedman JA, Wijdicks EF, Piepgras DG, Pichelmann MA, Toussaint LG 3rd, et al. Preoperative ventriculostomy and rebleeding after aneurysmal subarachnoid hemorrhage. J Neurosurg 2002;97:1042-1044

11. Roitberg BZ, Khan N, Alp MS, Hersonskey T, Charbel FT, Ausman Jl. Bedside external ventricular drain placement for the treatment of acute hydrocephalus. Br J Neurosurg 2001;15:324-327

12. Chung J, Lim YC, Suh SH, Shim YS, Kim YB, Joo JY, et al. Stent-assisted coil embolization of ruptured wide-necked aneurysms in the acute period: incidence of and risk factors for periprocedural complications. J Neurosurg 2014;121:4-11

13. Bruder M, Schuss P, Konczalla J, El-Fiki A, Lescher S, Vatter $H$, et al. Ventriculostomy-related hemorrhage after treatment of acutely ruptured aneurysms: the influence of anticoagulation and antiplatelet treatment. World Neurosurg 2015;84:1653-1659

14. Nguyen TA, Diodati JG, Pharand C. Resistance to clopidogrel: a review of the evidence. J Am Coll Cardiol 2005;45:1157-1164

15. Colwell AS, Reish RG, Kuter DJ, Damjanovic B, Austen WG Jr, Fogerty AE. Abdominal contouring procedures increase activity of the coagulation cascade. Ann Plast Surg 2012;69:129-133 